\title{
Unusual presentation of neurobrucellosis in Jeddah, Kingdom of Saudi Arabia
}

Mahdi A. Kanjo, MD, Hajar M. Ahmed, MBBS, Elaf A. Alnahari, MBBS.

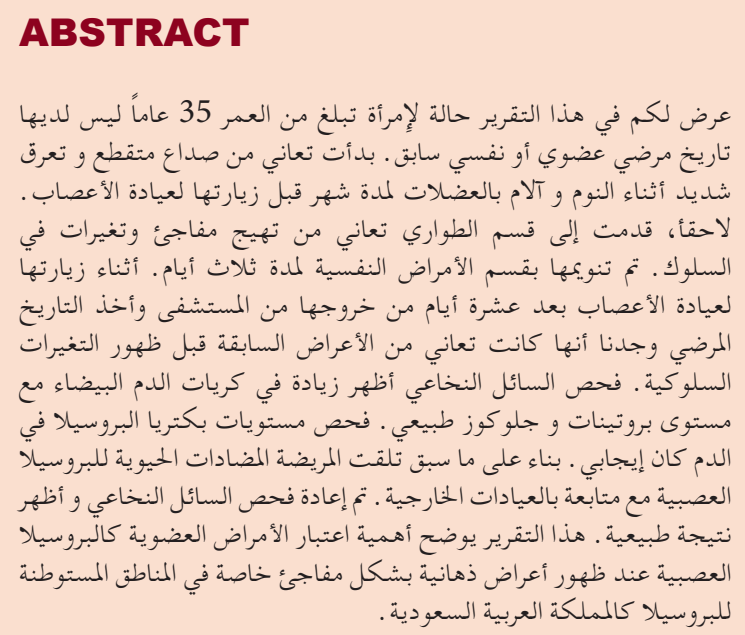

A 35-year-old female without any history of medical or psychiatric illness. One month prior to presenting to our clinic, she had intermittent headaches, night sweats, and muscle pain that went unnoticed by her. A week later, the patient developed agitation and behavioral changes. The patient was admitted through emergency room (ER) to the psychiatry unit for 3 days. During a detailed interview in the Neurology Clinic 10 days post discharge, she described the previously mentioned symptoms. Her cerebrospinal fluid analysis revealed leukocytosis, with normal protein and glucose, and positive brucella titers in blood. She received antibiotics for neurobrucellosis with frequent follow ups. Repeated spinal tap was reported normal. Her symptoms gradually improved. Neuropsychiatric manifestations are an unusual presentation of brucellosis. This case emphasizes the importance of considering an organic cause, such as neurobrucellosis, as a differential diagnosis in patients with new onset of unexplained neuropsychiatric symptoms in endemic countries like Kingdom of Saudi Arabia.

Neurosciences 2021; Vol. 26 (4):385-388 doi: 10.17712/nsj.2021.4.20210030

From the Department of Neurology (Kanjo); from the Department of Psychiatry (Ahmed), Dr.Soliman Fakeeh Hospital, Jeddah, and from the Department of Neurology (Alnahari), King Fahad hospital, Medina, Kingdom of Saudi Arabia.

Received 6th March 2021. Accepted 11th July 2021.

Address correspondence and reprint request to: Dr. Hajar M. Ahmed, Department of Psychiatry, Dr.Soliman Fakeeh Hospital, Jeddah, Kingdom of Saudi Arabia. E-mail: drhajarmohamed@gmail.com ORICID ID: https://orcid.org/0000-0003-2815-4610

$\mathrm{O}$ ne of the most common bacterial zoonoses in the world is brucellosis, it is endemic in Kingdom of Saudi Arabia (KSA) and the Mediterranean region and is also present in many other countries. ${ }^{1}$ Brucella can bypass the immune response and quickly spread across the body into multiple systems. ${ }^{2}$ Brucellosis can be acquired through several routes including direct inoculation through cuts and scratches (especially during handling animal tissues or secretions), inhalation of infected aerosols, through conjunctiva, or consumption of contaminated foods such as raw milk, unpasteurized milk cheeses, or raw meat. ${ }^{1}$ It has been also described to have human-to-human transmission including vertical and sexual transmission. ${ }^{3}$ The clinical presentations of brucellosis are diverse including systemic manifestations like fever, sweating, chills, and fatigue syndrome or localized such as epididymoorchitis and spondylodiscitis and it can present also by serious manifestations such as neurobrucellosis and endocarditis. ${ }^{3}$ Neurobrucellosis occurs in $0.5-25 \%$ of brucellosis cases and affects the central or peripheral nervous systems with serious complications. ${ }^{1}$ Various presentations of neurobrucellosis are encountered including psychiatric symptoms such as behavioral changes, psychosis, dementia, insomnia, restlessness

Disclosure. The authors declare no conflicting interests, support or funding from any drug company. 
and episodic crying and neurological manifestations such as meningitis, encephalitis, sensorial and motor abnormalities, polyradiculoneuritis, cranial nerve involvement, epilepsy, brain abscess, blurring of vision and optic neuritis, numbness, subarachnoid hemorrhage, and finally coma. ${ }^{1,4}$

Psychosis as a presentation of neurobrucellosis is a very unusual condition. There are only a few previous brucella reports with neuropsychiatric manifestations such as psychosis and abnormal behavior; therefore, we report a case of neurobrucellosis with agitation, abnormal behavior, and inappropriate speech.

Case Report. The informed consent was obtained from the patient for the case report. The timeline for this case report was summarized in Table 1. A 35-year-old female patient, married, has 2 children with no past history of psychiatric or medical diseases. The story of the patient started one and a half month prior to presentation to our clinic. She was having a severe on and off headache accompanied by blurred vision and numbness of the left hand. Moreover, she had severe night sweating, fatigue, and muscular pain especially in the neck and back. All previous symptoms went unnoticed by the patient as she didn't seek any medical advice during that time.

As stated by her husband, the patient started to have psychiatric symptoms for the first time one month prior to presentation to our neurology clinic when she started to develop acute agitation and behavioral changes in the form of shouting, rooming, confusion, and disorientation as well as inappropriate speech. The patient went to the Emergency Department of the nearest hospital, where some investigations were conducted including computed tomography (CT), brain magnetic resonance image (MRI), electrocardiography (ECG) and complete blood count $(\mathrm{CBC})$; the results were all reported to be within normal limits. Then, the patient was discharged home with the diagnosis of anxiety.

Two days post-discharge, the patient's symptoms still persisted (headache and behavioral changes). Therefore, she went to another hospital to ask for a second opinion. The patient was seen by a neurologist and then referred to psychiatry where she was admitted for 3 days as a case of acute confusional state for investigation at the psychiatry department. Upon investigations, $\mathrm{CBC}$, brain MRI, magnetic resonance venography, magnetic resonance angiography, brain CT were all reported as unremarkable. Patient was given Quetiapine, Citalopram, and Mirtazapine after which her symptoms improved. Patient could not remember what happened in the previous 3 days. On the third day patient was discharged in good condition as a case of conversion disorder.

Ten days post-discharge, the patient came to our neurology clinic for neurology opinion. Upon obtaining detailed history from the patient at the clinic we were able to get all the previously mentioned symptoms including headache, muscle pain, night sweat, etc.

Table 1 - Timeline.

\begin{tabular}{|c|c|}
\hline Dates & Past medical history \\
\hline & 35 years old female patient not known to have any medical or psychiatric illness. \\
\hline & Current illness \\
\hline May 2020 & Patient started to have on and off headache, numbness of the left hand, blurred vision, night sweats. \\
\hline 08 June 2020 & $\begin{array}{c}\text { Patient presented to ER with psychiatric symptoms in form of behavioral changes, inappropriate speech. Patient was admitted under } \\
\text { psychiatry department and was discharged in good condition after } 3 \text { days. }\end{array}$ \\
\hline \multirow[t]{2}{*}{23 June 2020} & The patient visited our neurology clinic for the first time where a detailed history and examination were made. \\
\hline & Physical examination \\
\hline \multirow[t]{2}{*}{23 June 2020} & within normal limits \\
\hline & Diagnostic evaluation \\
\hline 24 June 2020 & $\begin{array}{c}\text { Brucella antibody test in blood sample: positive for abortus and melitensis, CSF analysis: demonstrates Leukocytosis with predominant } \\
\text { lymphocytosis. }\end{array}$ \\
\hline \multirow[t]{2}{*}{28 June 2020} & Diagnosis was made. \\
\hline & Initial treatment \\
\hline \multirow[t]{2}{*}{28 June 2020} & Rifampicin $600 \mathrm{mg}$ OD, doxycycline $100 \mathrm{mg}$ BID, and moxifloxacin $400 \mathrm{mg}$ OD. \\
\hline & Ongoing treatment \\
\hline \multirow[t]{2}{*}{06 August 2020} & $\begin{array}{c}\text { Moxifloxacin was administered for } 6 \text { weeks, then stopped, and continued on rifampicin and doxycycline for a total of } 12 \text { weeks with a } \\
\text { frequent follow up with infectious disease and neurology clinics. }\end{array}$ \\
\hline & Final follow up \\
\hline 06 August 2020 & $\begin{array}{c}\text { Follow up spinal tap after one month reported normal cell count. Patient completed her antibiotic course for more } 6 \text { weeks with } \\
\text { significant improvement. }\end{array}$ \\
\hline
\end{tabular}


There was no history of fever, no history of recent travel, raw meat or unpasteurized diary product ingestion and also no history of animal contact. There is no history of similar condition in the family.

By adding amnesia and psychiatric symptoms that she experienced to the history, we suspected a Central Nervous System (CNS) infection namely, encephalitis and tuberculous meningitis.

Clinical findings. Upon physical examination, the patient was afebrile, conscious, and oriented to time, place, and person; the glasgow coma score was 15/15. She was not in distress, and her cognition, attention, and memory appeared normal. Dress and hygiene were appropriate. Affect was normal, and insight was good. Speech was fluent. Vision and pupils were normal, and there was no nystagmus on lateral and vertical gaze to either side. Smooth pursuit and saccadic eye movements were normal; extra-ocular movements were full; facial movements were symmetrical; gag response was intact. Power and muscle tone appeared to be within normal limits. Deep tendon reflexes were normal; plantar responses were both down going. Sensation to pin prick, touch, vibration, and position were normal. Coordination tests namely, finger to nose, heel to shin, and rapid alternating movements were intact. Posture, stance, and tandem walking were normal. Romberg test was negative. Gait was intact. Pull test was negative. The patient was admitted under neurology department for further investigations.

Diagnostic assessment. The WBC: 6.4 (normal range: $4.5-11 \quad\left(10^{3} \backslash \mathrm{uL}\right)$, lymphocytes: $16.1 \quad\left(10^{3} \backslash\right.$ $\mathrm{uL}$ ), neutrophils: $78.1\left(10^{3} \backslash \mathrm{uL}\right)$, Hemoglobin (HB): 11.1 (normal range: 11.7-15.5 gm/dl) microcytic hypochromic anemia, platelets: 281 (normal range 150-440 mcL), and C-reactive Protein (CRP): 4.69 $\mathrm{mg} / \mathrm{L}$ (normal range less than 10). Autoimmune screening Anti-nuclear antibody (ANA), Lupus anticoagulants, and anticardiolipin were all negative. Imaging: brain CT, MRI (Figure 1), and Magnetic Resonance Venography (MRV) were reported as unremarkable.

Cerebrospinal Fluid (CSF) analysis on initial presentation: Leukocytes 27 /microliter (normal range: less than 5), lymphocytes $100 \%$ (normal range: $\mathrm{Up}$ to $70 \%$ ), polymorph $0 \%$, protein $24.28 \mathrm{mg} / \mathrm{dl}$ (normal range: $15-45 \mathrm{mg} / \mathrm{dl}$ ), glucose $95.2 \mathrm{mg} / \mathrm{dL}$ (normal range: $40-70 \mathrm{mg} / \mathrm{dl}$ ), gram staining: negative, culture: negative, cytology: negative for malignant cells. Brucella antibody in CSF analysis was not performed as the sample were not enough. Film array was negative for meningeal panel. Brucella antibody test in blood: abortus 1:640, Melitensis 1:160. (positive ratio: single titer $\geq 1: 160$ ). The patient was diagnosed as a case of neurobrucellosis with neuropsychiatric presentation.

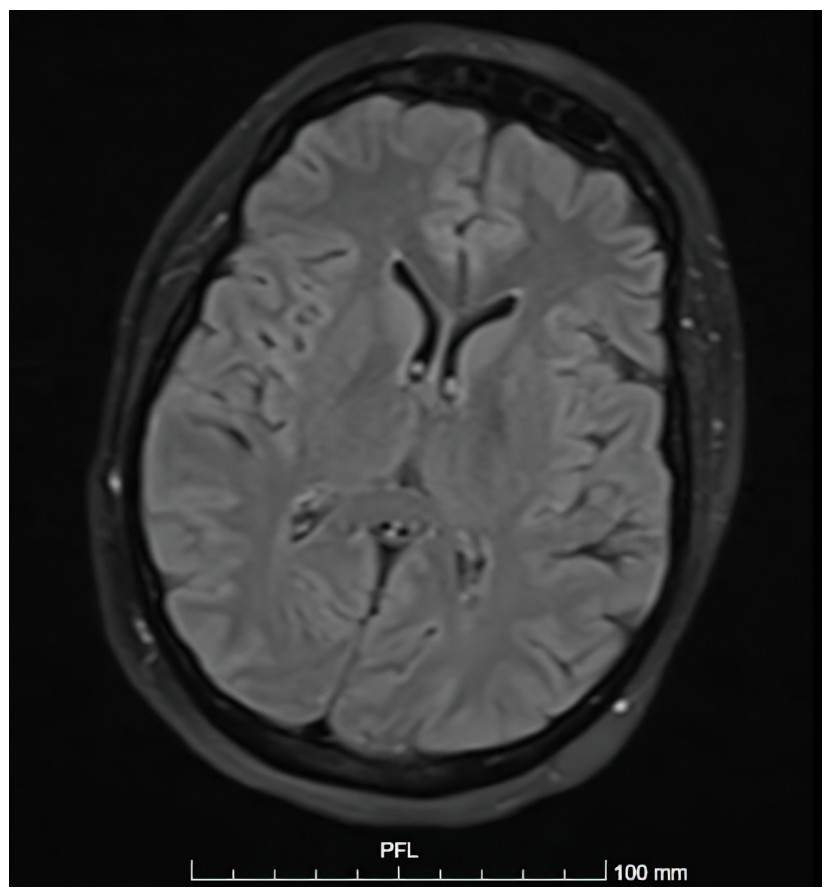

Figure 1 - MRI brain, axial cut, FLAIR, normal MRI of the brain.

Therapeutic intervention. The patient was started on Rifampicin $600 \mathrm{mg}$ OD, Doxycycline $100 \mathrm{mg}$ BID, and Moxifloxacin $400 \mathrm{mg}$ OD. Moxifloxacin was administered for 6 weeks, then stopped, and continued on Rifampicin and Doxycycline for a total of 12 weeks with a frequent follow up with infectious disease and neurology clinics.

Follow up and outcomes. Follow up spinal tap after one month reported normal cell count. Patient completed her antibiotic course for more 6 weeks with significant improvement in her symptoms.

Discussion. The pathophysiology behind CNS infection by brucella may involve allergic reactions or insufficient immune response or apoptosis. There are 2 known histological features; chronic granulomatous infection surrounding the vessels with multi-nucleated giant cells and lymphocytes, or large abscesses surrounded by astroglial scars that mimic a brain tumor. It can infect the brain, meninges, spinal cord, and cranial or peripheral nerves especially the vestibulocochlear nerve. ${ }^{4}$ The diagnosis of brucellosis is based upon the exclusion of several factors, including epidemiological data (possible exposure to the organism, residing in endemic areas). ${ }^{4}$ According to a previous study, one of the following diagnostic criteria is needed: presence of signs and symptoms suspecting neurobrucellosi; findings in imaging by cranial magnetic resonance imaging or 
computed tomography; pleocytosis with predominant lymphocytes and elevated protein concentration in CSF; positive culture or presence of brucella antibodies of a 4-fold or greater increase in the initial titer of CSF $\geq 1: 80$ using the standard tube agglutination test or a single high serum agglutination titer of $\geq 1: 160$; and reduction in the size of lesion or improvement of symptoms after an appropriate treatment of brucella. ${ }^{4,5}$

In this case, we made the diagnosis of neurobrucellosis based on the previous criteria because our patient had: a) typical symptoms of brucellosis (namely, night sweating, fatigue, and muscular pain) in addition to neuropsychiatric manifestations including headache, blurred vision, numbness, amnesia, agitation, behavioral changes, and inappropriate speech; b) CSF findings, namely, increase in lymphocytic count; and c) positivity of Brucella antibody titer in blood for 2 brucella species abortus 1:640 and Melitensis 1:160 (positive ratio: single titer $\geq 1: 160$ ).

The radiological findings of neurobrucellosis varies from a normal imaging study to various imaging anomalies, despite positive clinical results, which is the case with our patient who had normal CT and MRI results. ${ }^{6}$ The most common type of Brucella in the Middle East is melitensis followed by abortus, which were both positive in our patient; however, Brucella abortus had higher titer. ${ }^{7}$

Brucellosis affects multiple body systems with a wide range of clinical manifestations which makes the diagnosis very difficult. The acute manifestations of brucellosis are sometimes unspecific and may mimic certain neurological and psychiatric conditions. ${ }^{1}$ We have found in the literature approximately 18 case reports of brucellosis presented with psychosis. Similar to our case, there was a previously mentioned case report of brucellosis where the patient presented with abnormal behavior and hearing loss. ${ }^{8}$ Furthermore, in another case study, the patient was diagnosed as a case of mania for a long period of time before reaching the diagnosis of neurobrucellosis.?

Neurobrucellosis treatment plan should be fast and effective owing to the nature of the pathology. Treatment should include long-term antibiotics with adequate neuromeningeal diffusion, and it is typically based on a mixture of 2 or 3 antibiotics. ${ }^{2}$ The treatment for non-pregnant adults includes doxycycline $100 \mathrm{mg}$, twice daily, Rifampicin $600 \mathrm{mg}$, once daily, and thirdgeneration Cephalosporins $2.0 \mathrm{~g}$, IV twice-daily for the first 4-6 weeks and the duration should last for at least 3-6 months or until normalization of CSF. ${ }^{2,10}$

Patient perspective. The patient shared that this is her first time being introduced to this disease. Moreover, she expressed her concerns about the possibility of transmission of the infection to her children but we addressed her concerns. Finally, she was relieved to reach to a diagnosis for her condition and to start the proper treatment after going through a tough time between multiple hospitals and admission into the psychiatric ward as per patient words.

In conclusion, the differential diagnosis of CNS infections in general and neurobrucellosis specifically should be kept in mind in patients with unexplained neuropsychiatric symptoms or with no significant clinical or symptomatic improvement upon the treatment of other neuropsychiatric conditions; thus, these patients should be tested for neurobrucellosis to allow early diagnosis and care.

Acknowledgment. The authors gratefully acknowledge Dr. Sameh Al Haddad, Radiologist, for his interpretation of the brain MRI used in this case report. Also we would like to thank Falcon Scientific Editing (https:Iffalconediting.com) for proofreading the English language in this paper.

\section{References}

1. Bains R, Dahhan T, Belzowski A, Heinze ER, Wong AL, Clements PJ. An interesting case of neurobrucellosis mimicking neuropsychiatric lupus. Case Rep Rheumatol 2018; 2018: 9793535.

2. Zhao S, Cheng Y, Liao Y, Zhang Z, Yin X, Shi S. Treatment efficacy and risk factors of neurobrucellosis. Med Sci Monit 2016; 22: 1005-1012.

3. Tuon FF, Gondolfo RB, Cerchiari N. Human-to-human transmission of Brucella - a systematic review. Trop Med Int Health 2017; 22: 539-546.

4. Le Dudal M, Chrétien F, Jouvion G. Neurobrucellosis. Infections of the Central Nervous System 2020; 39.

5. Enani MA. Neurobrucellosis. In: Hasbun R, editors. Meningitis and Encephalitis. Springer International Publishing AG, part of Springer Nature; 2018. pp.97-106.

6. Erdem H, Senbayrak S, Meriç K, Batirel A, Karahocagil MK, Hasbun R, et al. Cranial imaging findings in neurobrucellosis: results of Istanbul-3 study. Infection 2016; 44: 623-631.

7. Algahtani H, Shirah B, Abdulghani D, Farhan R, Algahtani R. Occupational neurobrucellosis mimicking a brain tumor: a case report and review of the literature. Case Rep Infect Dis 2017; 2017: 1434051.

8. Shah IA, Kawoos Y, Sanai BA, Rabyang S, Banday D. Neurobrucellosis presenting as acute psychosis. J Neurosci Rural Pract 2018; 9: 644-646.

9. Akcay Hİ, Kurtuncu M, Celik SY, Gunduz T, Eraksoy M. Neurobrucellosis presenting with mania. Acta Neurol Belg 2017; 117: 373-375.

10. Patra S, Kalwaje Eshwara V, Pai AR, Varma M, Mukhopadhyay C. Evaluation of clinical, diagnostic features and therapeutic outcome of neurobrucellosis: a case series and review of literature. Int J Neurosci 2021; 1-11. 